\author{
L. Peruzzi, G. Gestri \& B. Pierini
}

\title{
Morphological variation in Astragalus muelleri (Fabaceae) from Balkan and Italian populations
}

\begin{abstract}
Peruzzi, L., Gestri, G. \& Pierini, B.: Morphological variation in Astragalus muelleri (Fabaceae) from Balkan and Italian populations. - Fl. Medit. 24: 233-238. 2014. - ISSN: 1120-4052 printed, 2240-4538 online.

A morphometric study aimed at comparing Italian and Balkan populations of Astragalus muelleri is carried out. Based on univariate (ANOVA, Tukey HSD test) and multivariate (DA) analysis results, a new subspecies to accommodate the Italian populations (A. muelleri subsp. etruscus subsp. nov.) is described. This subspecies is slightly distinct from the typical Balkan populations for its fewer and larger flowers, producing shorter fruits.
\end{abstract}

Key words: Croatia, flora, taxonomy, Tuscany.

\section{Introduction}

Astragalus muelleri Steud. \& Höchst. (Fabaceae) was originally described from the Island of Krk (Veglia in classical times), N Croatia (Höchstetter 1827: 72, "auf Veglia"). It is a species linked to rocky calcareous substrates at low altitudes, occurring on the coasts and islands of western Balkans (from Croatia to Montenegro), with disjunct localities in Tuscany, Central Italy (Nikolić 2004 onwards; Pignatti 1982). The plant was also quoted for Abruzzo (Cesati 1873; Greco \& Petriccione 1989), but herbarium documentation is missing and the records are very likely wrong (Conti \& al. 2005; F. Conti, F. Bartolucci pers. comm.). The Italian populations occur in two areas: a first one, smaller, in N Tuscany (Monte Pisano; Pierini \& al. 2009 and literature cited therein), a second one, larger, in S Tuscany (Monte Amiata area; Selvi \& Fiorini 1994; Selvi 2010). Given the noteworthy disjunction of these populations from the Balkan ones, we decided to carry out a comparative study.

\section{Materials and Methods}

Given that in Tuscany 2 main population groups occur, we selected for comparison two further populations from the Balkan species range in Croatia: a first one just in front of the 
locus classicus (Senj), and a second one more southerly (Obrovac). We examined a total of 37 herbarium specimens. Those from Monte Pisano (M.PISANO, 8), Obrovac (OBROVAC, 7) and Senj (SENJ, 7) were collected in ad hoc field expeditions and deposited in PI, while 15 specimens from Monte Amiata (AMIATA) area were studied in FI herbarium (see Specimina visa). The following 12 quantitative characters were considered: 1) Plant size $(\mathrm{cm})$; 2) Flowers number per flowering stem; 3) Calyx length (mm); 4) Calyx teeth length (mm); 5) Banner length (mm); 6) Wings length (mm); 7) Keel length (mm); 8) Fruit length (mm); 9) Fruit width (mm); 10) Foliar segments number; 11) Foliar segment length (mm, the largest in a sample); 12) Foliar segment width ( $\mathrm{mm}$, the largest in a sample). The data were subjected to univariate and multivariate statistics by means of PAST 3.01 software (Hammer 2013; Hammer \& al. 2001). For each character the variation among the 4 population groups and among Balkan and Italian populations was tested. The variability of the analyzed morphological characters was described by standard statistical parameters (mean, standard deviation, minimum, maximum, 10th and 90th percentiles). Furthermore, the variables were processed singularly, by means of ANOVA (after check of Levene statistics) with Tukey HSD test for comparison of the same variable among pairs of the four Operational Taxonomic Units (OTU). To test the overall discrimination power of the considered characters alone, classificatory Discriminant Analysis (DA) was carried out. DA is an identification optimization procedure based on the probability of identification (e.g., Geisser's classification probabilities) and was performed on the dataset of 12 continuous variables and 37 accessions. A priori classification (Henderson 2006) was used to test whether the circumscription of the four population groups (used as groups in the Discriminant Analysis), also fits with the quantitative data. The same was done for two larger groups, defined according to their regional provenance (Italy vs. Balkans). This model assumes that each OTU is a discrete unit. The Discriminant Analysis developed predictive discriminant functions for the groups, which were, in turn, subsequently applied to the accessions in each group. The model used a priori probability equal for each group, when classifying the accessions.

\section{Results}

The univariate relationships among the four considered OTUs are represented in Table 1. Most of the characters, taken singularly, show no statistically significant differences among the studied OTU, with the exception of the character \#2 (flowers number), \#7 (keel length), and \#8 (fruit length). Concerning these characters, for \#2 there is no difference between SENJ, M.PISANO and OBROVAC (Tukey HSD test with $\mathrm{p}$ values ranging between 0.15 and 0.99 ), while moderately significant differences occur between M.PISANO and AMIATA ( $\mathrm{p}=0.013$ ), and significant differences occur between the other OTU pairs $(p=0.00)$. For \#7 there is no difference between most OGUs (Tukey HSD test with $p$ values ranging between 0.07 and 0.55 ), with the exception of a moderately significant difference occurring between SENJ and AMIATA $(\mathrm{p}=0.03)$, and a significant difference between SENJ and M.PISANO $(\mathrm{p}=0.003)$. For \#8 there is no difference between M.PISANO and AMIATA $(\mathrm{p}=0.76)$, while a moderately significant difference occurs between AMIATA and OBROVAC ( $\mathrm{p}=0.023)$, and significant differences occur between the other OTU pairs (Tukey HSD test with p values ranging between 0.000 and 0.006 ). 
Table 1. Morphological features for each studied population, and for grouped Italian and Balkan populations. Values are expressed as 10-90 percentiles, with extremes in brackets, and as mean \pm standard deviation. $*=$ characters for which ANOVA evidenced significant differences.

\begin{tabular}{|c|c|c|c|c|}
\hline Character & M. Pisano & M. Amiata & Senj & Obrovac \\
\hline & \multicolumn{2}{|c|}{ Italy } & \multicolumn{2}{|c|}{ Croatia } \\
\hline \multirow[t]{4}{*}{ Plant size $(\mathrm{cm})$} & $(21-) 21.7-30.9(-33)$ & $25-36.2(-39)$ & $(19-) 20.2-30.4(-31)$ & $20-28.6(-31)$ \\
\hline & $25.38 \pm 4.10$ & $29.53 \pm 4.16$ & $25.57 \pm 4.83$ & $23.57 \pm 4.03$ \\
\hline & \multirow{2}{*}{\multicolumn{2}{|c|}{$\begin{array}{c}(21-) 23.2-34.6(-39) \\
28.09 \pm 4.79\end{array}$}} & \multicolumn{2}{|c|}{$(19-) 20-30.7(-31)$} \\
\hline & & & \multicolumn{2}{|c|}{$24.57 \pm 4.4$} \\
\hline \multirow[t]{4}{*}{ Flowers number* } & $\begin{array}{l}(7-) 9.8-22.8(-27) \\
\end{array}$ & $8-13.6(-16)$ & $(15-) 16.8-28.4(-29)$ & $(15-) 17.4-25.8(-27)$ \\
\hline & $16.88 \pm 6.1$ & $10.67 \pm 2.47$ & $21.29 \pm 5.34$ & $21.71 \pm 4.07$ \\
\hline & \multicolumn{2}{|c|}{$(7-) 8-18.8(-27)$} & \multicolumn{2}{|c|}{$(15-) 15.9-27.7(-29)$} \\
\hline & \multicolumn{2}{|c|}{$12.83 \pm 4.98$} & \multicolumn{2}{|c|}{$21.5 \pm 4.57$} \\
\hline \multirow[t]{4}{*}{ Calyx length $(\mathrm{mm})$} & $(8-) 8.35-11$ & $(7.5-) 7.9-11$ & $7.5-8.9(-9.5)$ & $(5.5-) 5.8-9.5$ \\
\hline & $9.44 \pm 1.11$ & $9.22 \pm 1.28$ & $8.14 \pm 0.69$ & $8.07 \pm 1.69$ \\
\hline & \multicolumn{2}{|c|}{$(7.5-) 8-11$} & \multicolumn{2}{|c|}{$(5-) 6.45-9.5$} \\
\hline & \multicolumn{2}{|c|}{$9.32 \pm 1.17$} & \multicolumn{2}{|c|}{$8.11 \pm 1.24$} \\
\hline \multirow{4}{*}{$\begin{array}{l}\begin{array}{l}\text { Calyx } \\
(\mathrm{mm})\end{array} \\
\end{array}$} & $(0.7-) 0.77-1.29(-1.5)$ & $1-1.5$ & $(0.5-) 0.62-1.08(-1.08)$ & $(0.5-) 0.6-1(-1.1)$ \\
\hline & $1.03 \pm 0.24$ & $1.19 \pm 0.24$ & $0.87 \pm 0.24$ & $0.86 \pm 0.21$ \\
\hline & \multicolumn{2}{|c|}{$(0.7-) 0.92-1.5$} & \multicolumn{2}{|c|}{$(0.5-) 0.56-1.07(-1.2)$} \\
\hline & \multicolumn{2}{|c|}{$1.11 \pm 0.25$} & \multicolumn{2}{|c|}{$0.86 \pm 0.22$} \\
\hline Banner length (mm) & $(17-) 18.05-21.3(-22)$ & $(17-) 17.8-20.4(-22)$ & $(15-) 16.5-20$ & $16-20.8(-22)$ \\
\hline & $19.63 \pm 1.53$ & $19.17 \pm 1.54$ & $18.36 \pm 1.84$ & $18.86 \pm 2.19$ \\
\hline & $(17-) 17$ & $21.4(-22)$ & $(15-) 1$ & $20(-22)$ \\
\hline & 19 & 1.51 & 18.6 & \pm 1.96 \\
\hline Wings length $(\mathrm{mm})$ & $(16-) 16.35-19$ & $15-15.8(-19)$ & $(13-) 14.2-18$ & $15-17.4(-18)$ \\
\hline & $17.81 \pm 1.07$ & $16.89 \pm 1.45$ & $16.21 \pm 1.78$ & $16.29 \pm 1.25$ \\
\hline & & $6-19$ & $(13$ & $5-18$ \\
\hline & 17. & 1.33 & 16.2 & \pm 1.47 \\
\hline Keel length $(\mathrm{mm})^{*}$ & $(14.5-) 14.85-17.3(-18$ & $(14-) 14.8-16.2(-17)$ & $(12-) 12.6-15$ & $14-15.4(-16)$ \\
\hline & $16.06 \pm 1.18$ & $15.50 \pm 0.87$ & $14 \pm 1.15$ & $14.71 \pm 0.77$ \\
\hline & $(14-) 1$ & $-17(-18)$ & $(12-) 13$ & $-15(-16)$ \\
\hline & 15 . & 1.03 & & \pm 1 \\
\hline Fruit length $(\mathrm{mm})^{*}$ & $14-17.4(-19.5)$ & $(10-) 10.4-18.2(-19)$ & $(17-) 17.6-22$ & $(9-) 9.6-11.7(-12)$ \\
\hline & $15.43 \pm 1.92$ & $14.28 \pm 3.35$ & $20 \pm 1.98$ & $10.64 \pm 0.99$ \\
\hline & $(10-) 11$. & $8.5(-19.5)$ & $(9-) 10.1$ & $21.85(-22)$ \\
\hline & 14 & 2.79 & 15.3 & \pm 5.08 \\
\hline Fruit width $(\mathrm{mm})$ & $(2.5-) 2.56-3.2(-3.5)$ & $2.5-3.5$ & $3-3.5$ & $(2.5-) 2.8-3.5$ \\
\hline & $2.91 \pm 0.33$ & $2.98 \pm 0.44$ & $3.14 \pm 0.24$ & $3.07 \pm 0.34$ \\
\hline & & 3.5 & $(2.5$ & $3-3.5$ \\
\hline & & 0.38 & 3.1 & 0.29 \\
\hline segments & $(13-) 13.7-15.6(-17)$ & $11-15$ & $(12-) 12.6-19.2(-21)$ & $13-15.8(-17)$ \\
\hline number & $14.75 \pm 1.16$ & $13 \pm 1.63$ & $15.86 \pm 3.08$ & $14.71 \pm 1.38$ \\
\hline & & $(-17)$ & $(12-) 13$ & $7.7(-21)$ \\
\hline & 13. & 1.66 & 15.2 & \pm 2.37 \\
\hline Foliar segment length & $(8-) 8.35-13.65(-14)$ & $9-12.2(-14)$ & $(8.5-) 9.1-12.8(-14)$ & $(6-) 6.6-12.5(-14)$ \\
\hline$(\mathrm{mm})$ & $11.25 \pm 2.27$ & $10.7 \pm 1.49$ & $10.57 \pm 1.90$ & $9.93 \pm 2.71$ \\
\hline & $(8-) 8.8$ & $3.65(-14)$ & $(6-) 7.4$ & $13.4(-14)$ \\
\hline & 10. & $=1.83$ & 10.2 & \pm 2.27 \\
\hline Foliar segment width & $(0.5-) 0.71-1.86(-2)$ & $1-1.55(-2)$ & $(0.7-) 0.88-1.7(-2)$ & $(0.5-) 0.68-1.14(-1.2)$ \\
\hline$(\mathrm{mm})$ & $1.19 \pm 0.5$ & $1.37 \pm 0.32$ & $1.21 \pm 0.42$ & $0.87 \pm 0.23$ \\
\hline & $\overline{(0.5-) 0}$ & $-1.86(-2)$ & $(0.5-) 0$. & $-1.41(-2)$ \\
\hline & 1.2 & 0.41 & 1.0 & 0.37 \\
\hline
\end{tabular}


When a Discriminant Analysis is carried out, with the four populations recognized as $a$ priori groups, correct classification results in $83.78 \%$ (62.16\% if jackknifed), with possible confusions mostly due to the overlap of M.PISANO with AMIATA and only partially with OBROVAC (Fig. 1). Indeed, when a Discriminant Analysis is carried out by recognizing Italian and Balkan populations as a priori groups, the correct classification percentage increases to $94.59 \%$ (72.97\% if jackknifed), with only 1 Italian individual incorrectly classified in the Balkan group and vice versa.

\section{Discussion}

Our results show a certain degree of morphological variation within A. muelleri. In particular, the Italian populations are slightly differentiated from the Balkan ones. Taking in consideration also the relevant geographical separation, i.e. more than $200 \mathrm{Km}$ of territories including the Adriatic Sea (Fig. 2), we think that taxonomic recognition of this variation at subspecies rank is appropriate, according to the following taxonomic setting:

Astragalus muelleri Steud. \& Höchst., Flora 10: 72. 1827.

Lectotype (designated by Podlech 1999: 184) - Ins. Veglia, May 1826, Müller s.n. (TUB, isolectotypes in $\mathrm{K}, \mathrm{W})$.

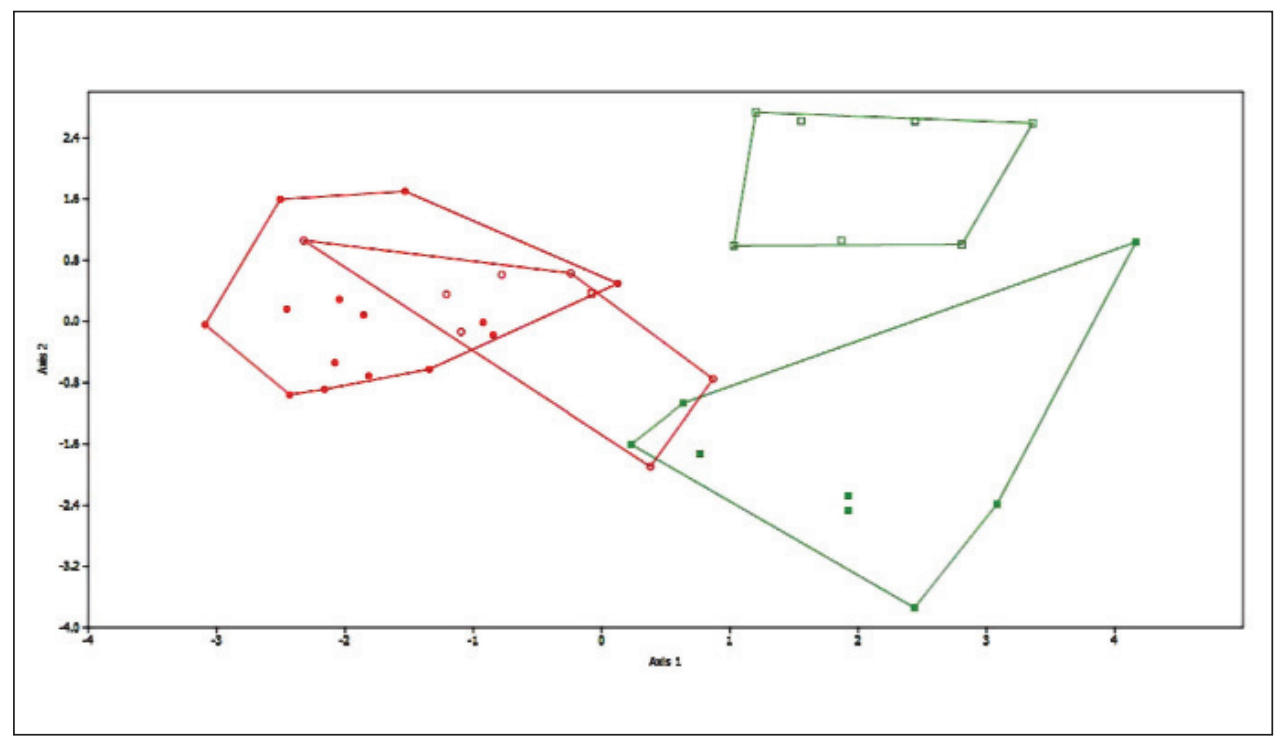

Fig. 1. DA based on the 12 considered morphological characters, with 4 a priori identified groups corresponding to the OTUs within Astragalus muelleri. Filled dots: AMIATA, empty dots: M.PISANO. Empty squares: SENJ, filled squares: OBROVAC. The characters which much contributed to the discrimination were (with decreasing absolute correlation values between variables and discriminant functions) on Axis 1: Flower number, Plant size, $n^{\circ}$ foliar segments; on Axis 2: Fruit length, Flower number, $n^{\circ}$ foliar segments. 


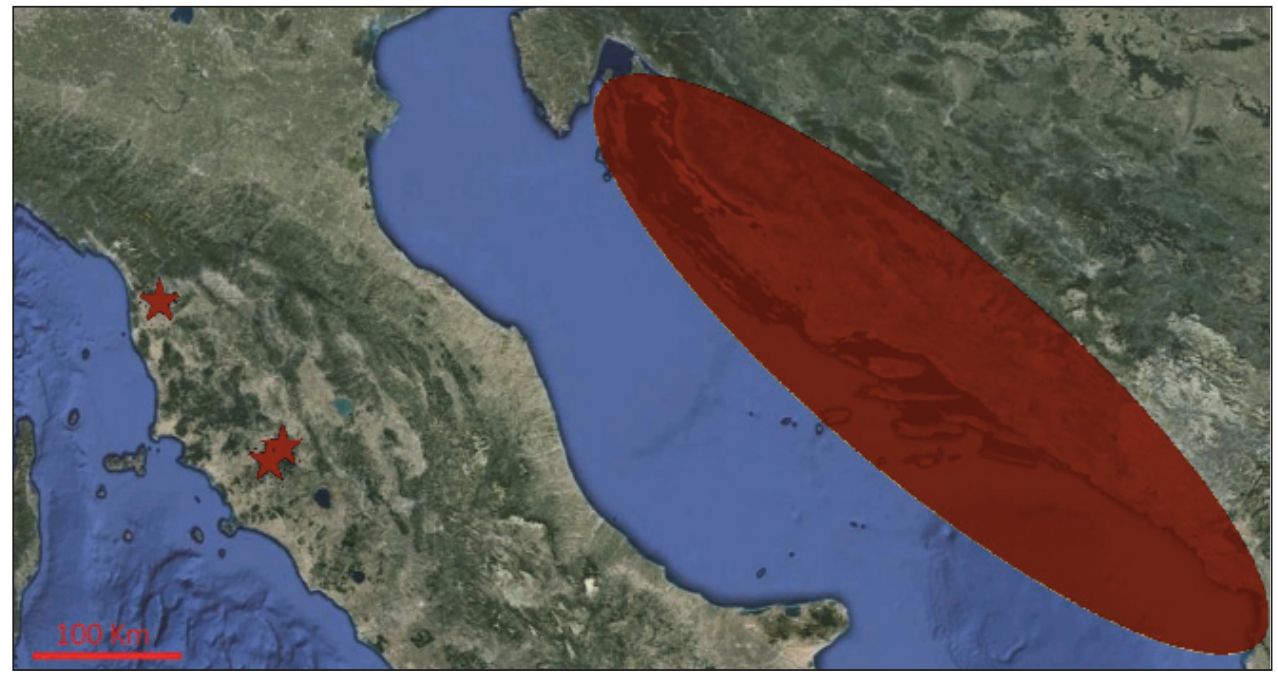

Fig. 2. Geographic distribution of Astragalus muelleri subsp. muelleri (oval), and A. muelleri subsp. etruscus subsp. nov. (stars).

\section{subsp. muelleri}

Distribution - Western Balkan coasts and islands.

Chromosome number - unknown.

Specimina visa - CROATIA. Croazia, Obrovazzo (Obrovac), ambienti aridi e sassosi, UTM: 33T 552329.4896457, 117 m, 28 April 2013, L. Peruzzi, G. Gestri, B. Pierini s.n. (PI); Croazia, Segna (Senj), ambienti aridi e rocciosi, UTM: 33T 49400000.4979447, 47 m, 28 April 2013, L. Peruzzi, G. Gestri, B. Pierini s.n. (PI).

subsp. etruscus Peruzzi, Gestri \& Pierini subsp. nov.

It is distinct from subsp. muelleri for its smaller flower number [(7-)8-19(-27) vs. (15-)16-28(-29)], larger flowers [e.g. keel (14-)14.8-17(-18) vs. (12-)13.3-15(-16) mm long] and shorter fruits [(10-) $11.5-18.5(-19.5)$ vs. (9-)10.15-21.85(-22) mm long].

Holotype - Agro di Seggiano, Piagge, loc. Calanche, suffruticeti sui pendii calanchivi argilloso-calcarei verso il T. Vivo, 500 m, diffuso, 11 July 2009, F. Selvi 3195 (FI).

Distribution - Central Italy, Tuscany (Monte Pisano and Monte Amiata area).

Chromosome number $-2 n=16$ (Selvi \& Fiorini, 1994).

Specimina visa altera (paratypes) - ITALY, TUSCANY. in saxosis calcareis Montis Pisani juxtus [...] S. Juliani, May 1841, P. Savi s.n. (FI); tra Seggiano e Castel del Piano, 28 May 1853, F. Parlatore (FI); in lapidosis prope pagum M. Pescali, 4 May 1882, G. Arcangeli s.n. (FI); Loc. Etruria, Prov. Grosseto: Montepescali, locis saxosis collium, oppidi meridiem versus sitis, Medicaginis prostratae var. pubescentis socius, alt. $320 \mathrm{~m}$. circ., solo argilloso-calcareo, 28 May 1911, A. Fiori s.n. (FI); Toscana, agro di Seggiano in loc. "Le Piagge", boscaglie rade e scarpate rupestri lungo la strada per il Vivo, calcare, 500 m, 13 July 2001, F. Selvi s.n. (FI); Grosseto, 
Poggio di Moscona, garighe rupestri su suolo calcareo sul versante sud, m 200 circa, 1 May 2004, F. Selvi 1720 (FI); Monte Pisano, Sella di Catro (Lucca), 300-400 m, 3 May 2013, B. Pierini, L. Peruzzi s.n. (PI).

\section{References}

Cesati, V. 1873: Congresso degli alpinisti in Chieti. Elenco delle piante raccolte da diversi fra li botanici che presero parte all'escursione sul gruppo della Maiella e del Morrone. - Boll. CAI 7: 157-187.

Conti, F., Abbate, G., Alessandrini, A., Blasi, C. (Eds.) 2005: An annotated checklist of the Italian vascular flora. - Roma.

Greco, S. \& Petriccione, B. 1989: La cartografia della vegetazione nella definizione della qualità dell'ambiente: il caso di Cocullo (AQ). - Not. Fitosoc. 24(1988): 63-98.

Hammer, Ø. 2013: PAST 3.01. - http://folk.uio.no/ohammer/past [accessed July 2014].

—, Harper, D.A.T., Ryan, P.D. 2001: PAST: Paleontological Statistics sofware package for education and data analysis. - Paleontol. Electr. 4(1): 1-9.

Henderson, A. 2006: Traditional morphometrics in plant systematics and its role in palm systematics. - Bot. J. Linn. Soc. 151: 103-111. doi: 10.1111/j.1095-8339.2006.00526.x

Höchstetter, H., 1827: Ueber die Leistungen des botanischen Reisenvereins im jahr 1826. - Flora 10: $65-74$.

Nikolić, T., 2004 onwards: Flora Croatica Database - Vascular plants Taxonomy \& Bibliography of Croatian Flora. -Available on the internet at http://hirc.botanic.hr/fcd/ [accessed 23 August 2014].

Pierini, B., Garbari, F., Peruzzi, L., 2009: Flora vascolare del Monte Pisano (Toscana nord-occidentale). - Inform. Bot. Ital. 41(2): 147-213.

Pignatti, S. 1982: Flora d'Italia, 1. - Bologna.

Podlech, D. 1999: Typification of Astragalus species III. (Leguminosae). - Sendtnera 6: 175-191.

Selvi, F. 2010: A critical checklist of the vascular flora of Tuscan Maremma (Grosseto provicne, Italy). - Fl. Medit. 20: 47-139.

-, Fiorini, G. 1994: Aspetti fitogeografici e cariologici della flora del Poggio di Moscona (Grosseto). - Atti Soc. Tosc. Sci. Nat., Ser. B, 101: 145-164.

Addresses of the authors:

Lorenzo Peruzzi ${ }^{1}$, Giovanni Gestri ${ }^{2} \&$ BrunelloPierini ${ }^{3}$,

${ }^{1}$ Dipartimento di Biologia, Università di Pisa, via Luca Ghini 13, 56126 Pisa, Italy, e-mail: lorenzo.peruzzi@unipi.it

${ }^{2}$ via Bonfiglioli 30, 59100, Prato, Italy, e-mail: ggestri@alice.it

${ }^{3}$ via Zamenhof 2, 56127 Pisa, Italy, e-mail: brunellopierini1@virgilio.it 